\title{
How to Build a Two-Photon Microscope with a Confocal Scan Head
}

\author{
Volodymyr Nikolenko and Rafael Yuste
}

This article provides practical guidelines for the conversion of a standard confocal microscope into a two-photon microscope. This conversion enables the investigator to have access to two-photon microscopy without the large budget necessary to purchase a commercial instrument. Two-photon fluorescence microscopy allows deep-tissue imaging in highly scattering preparations and long-term imaging of live tissue without the photodamage that is caused by out-of-focus light. It is, therefore, an essential tool for imaging cells under physiologically relevant conditions such as acute or cultured brain slices or in vivo.

IMAGING SETUP

The particular components described here are those in use at the time of the writing of this article.

The key component of the system is a femtosecond-pulsed laser that can generate reasonable power in the near-infrared (NIR) spectral region needed for convenient practical imaging (average power $>50 \mathrm{~mW}$ ). Our current setup uses the Chameleon laser from Coherent, Inc. This is a fully automated turnkey laser, which can be tuned to any wavelength between 690 and $1080 \mathrm{~nm}$.

Optical Table

The laser beam is delivered to a modified Olympus FluoView confocal laser-scanning system through the set of optical elements on the optical table (Fig. 1). The optical elements are intermediate mirrors, a spatial filter, a retardation wave plate, and a Pockels cell. BB1-E02 dielectric mirrors from Thorlabs, Inc. are used as intermediate mirrors. These reflect $>99 \%$ of light between 700 and $1150 \mathrm{~nm}$ at a $45^{\circ}$ angle of incidence for all polarizations and do not introduce additional group velocity dispersion of ultrafast pulses. An optical spatial filter, which has two plano-convex lenses and a pinhole in the focus of the first lens, acts as a simple telescope. It is used to restore a smooth Gaussian profile to the intensity of the laser beam cross section and also to modify the beam size to ensure proper overfilling of the back aperture of the microscope objective (Tsai et al. 2002). A retardation wave plate $(\lambda / 2$ or $\lambda / 4)$ is used for complex experiments in which the polarization of the scanning laser beam must be controlled.

Adapted from Imaging: A Laboratory Manual (ed. Yuste). CSHL Press, Cold Spring Harbor, NY, USA, 2011.

(C) 2013 Cold Spring Harbor Laboratory Press

Cite this article as Cold Spring Harb Protoc; 2013; doi:10.1101/pdb.ip075135 


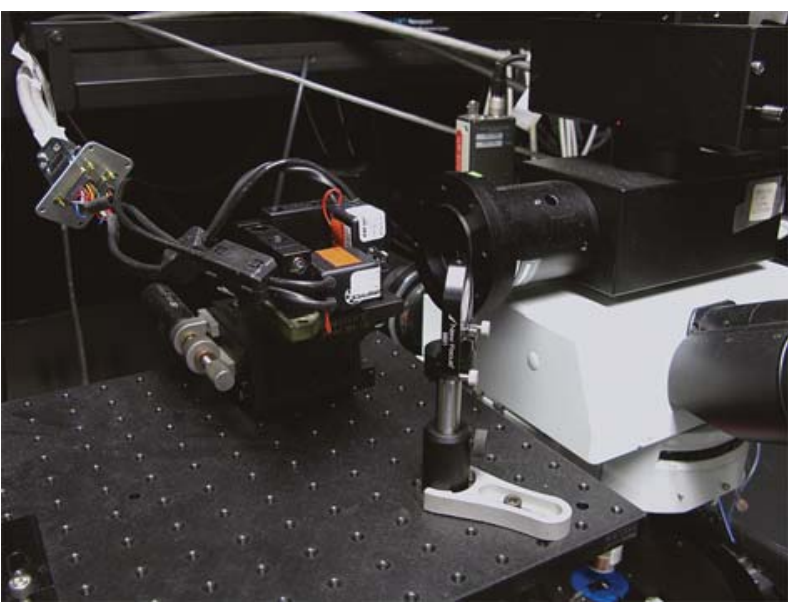

FIGURE 1. (A) Optical design of the instrument. Major components: (1) NIR femtosecond-pulsed laser; (2) Pockels cell modulator; (3) system of lenses, which works as a spatial filter and a beam expander ( $1.2 \times$ in our case); (4) periscope mirrors (which deliver the laser beam from the optical table level to the scanning box of the FluoView, which is raised for the upright microscope); (5) modified Olympus FluoView scanning unit; (6) external photomultiplier tube (PMT) detector for the 2 P-fluorescence signal, attached to the camera port of the microscope; (7) second external PMT attached to the microscope through a custom-made adapter; (8) intermediate signal amplifier for matching the dynamic range of the signal source (PMT) and the FluoView data acquisition module. $(B)$ A photograph of the modification of the FluoView confocal system (element 5 in $A$ ): The original galvanometer mirrors are completely removed from the Olympus scanning unit and are placed on a generic breadboard in front of the pupil transfer (scan) lens.

Pockels Cell

A 350-160 Pockels cell, a nonlinear optical modulator from Conoptics, is included to allow the dynamic modulation of laser light intensity with excellent contrast $(>200: 1)$ and submicrosecond temporal resolution. The temporal resolution of the Pockels cell is limited, in practical terms, only by the electronics of the high-voltage driver. The model in our current setup (275 linear amplifier from Conoptics) can work in a DC-8-MHz modulation range (thus providing 125 -nsec temporal resolution). 


\section{Scanning Microscope}

A BX50WI Olympus upright scanning microscope is coupled to a modified FluoView scanning unit. The Olympus FluoView platform relieves the need for a separate, custom-built scanning system and software package (Majewska et al. 2000).

The scanning box contains only one essential component: a set of galvanometer mirrors, which steer the laser beam and scan the image (see Fig. 1B). The scanning box is optically linked to the infinity-corrected BX50WI microscope through a pupil transfer lens (or scan lens), which is part of the original FluoView system. This lens, together with the tube lens of the microscope (original part of the microscope), forms a telescope, which provides collimated light for the infinity-corrected objective lens (see optical scheme in Fig. 1). This telescope also approximately images the scanning mirrors onto the back aperture of the objective. This minimizes the movement of the laser beam at the back aperture, thus reducing variation of laser power at the sample. The laser beam is reflected downward by a short-pass dichroic mirror (650DCSP or similar from Chroma Technology) placed inside the standard trinocular tube of the Olympus microscope.

\section{Fluorescence Detection}

In the case of two-photon absorption, excitation of fluorescence is essentially limited to the diffractionlimited spot in the focal plane. This provides the 3D sectioning characteristic of two-photon microscopy. Because fluorescence from the excited region irradiates in all directions, it is important to use a high-numerical-aperture objective to collect as many fluorescence photons as possible. Our system uses an external photomultiplier tube (PMT) as a detector. The tube is mounted on the camera port of the microscope's trinocular tube-along with the dichroic mirror mentioned above, which transmits visible fluorescent light collected by the objective. An additional infrared (IR)-blocking filter (et700sp$2 \mathrm{p} 8$ from Chroma Technology) is placed in front of the PMT to filter out residual IR fluorescent light reflected from the excitation path. The external PMT could also be positioned right next to the objective (see schematic in Fig. 1). In this case, the PMT would have to be mounted via a custommade adapter with a long-pass dichroic mirror, which transmits excitation IR light and reflects visible fluorescence to the detector. Positioning the PMT on top of the objective improves light collection efficiency but compromises the convenient positioning of the micromanipulators.

By choosing additional appropriate dichroic mirrors, it is possible to use multiple channels of fluorescence imaging. Placing additional band-pass filters in front of the detectors can also efficiently separate the emissions of different fluorescent dyes. The Olympus FluoView data acquisition board comes already equipped with two independent input channels.

The PMT used in our instrument is the H7422P-40 assembly from Hamamatsu, which comprises a GaAsP, a head-on PMT, a high-voltage power supply, and a thermoelectric cooler. This PMT provides a current signal that is proportional to the light intensity. The signal is fed into a current preamplifier (SR570, Stanford Research Systems [SRS]) and then into the Olympus FluoView data acquisition board. The SR570 has variable gain and adjustable high/low-pass filters and can be controlled through a user-friendly computer interface. The preamplifier is very convenient for proper signal conditioning because it allows proper filling of the dynamic range of the FluoView signal analog-to-digital converter, something crucially important for imaging weak signals and quantitative measurements. The standard Olympus FluoView software is used in the scanning mode for signal acquisition and reconstruction of a digital image. For calibration curves of the available dynamic range of the Olympus FluoView signal inputs, see Nikolenko et al. (2003).

The full description of the practical changes in the standard Olympus FluoView confocal system can be found in Majewska et al. (2000) and Nikolenko et al. (2003). A brief summary of the necessary modification is presented here. 
1. Install the pulsed femtosecond NIR laser.

2. Build the external optical pathway from the laser source to the laser-scanning microscope with the optional spatial filter and the Pockels cell.

3. Modify the scanning unit of a confocal laser-scanning microscope for scanning by the IR beam from the external laser source. This essentially requires drilling a hole in the back of the scanning unit and replacing the internal dichroic mirror with an IR-reflecting mirror (see the full list of Olympus FluoView modifications in Majewska et al. 2000 and Nikolenko et al. 2003). An alternative and more radical approach to drilling the hole in the original FluoView scan unit is to remove the scanning mirrors with their control cables altogether and to mount them on a separate optical breadboard as shown in Figure 1B.

4. Place a short-pass dichroic mirror into the trinocular tube of the optical microscope, and install the external detector (PMT) on the camera-imaging port of the trinocular tube.

5. Connect the detector signal output to the data acquisition input of the confocal system through the intermediate signal amplifier.

\section{ADVANTAGES AND LIMITATIONS}

The two-photon system described here, based on the Olympus FluoView confocal system, successfully combines a customized homemade system with a reliable commercial instrument. Although tailormade for the chosen application, the individual elements of a homemade system can be difficult to maintain in optimal working condition. However, recent advances are easing this problem. For example, the appearance on the market of turnkey femtosecond-pulsed tunable lasers, such as the Chameleon, eliminates the need for realignment of the optical path. Potential users, however, should be aware of the correct procedures for cleaning optical surfaces that are exposed to dust, changes in humidity, etc., and should follow proper laser safety guidelines.

The custom-made two-photon microscope described here has been used successfully for long-term imaging of the action potential activity in large $(>1000)$ populations of neocortical neurons in acute brain slices loaded with $\mathrm{Ca}^{2+}$ fluorescent indicators such as Fura-2-acetoxymethyl ester (Fura-2AM) (Cossart et al. 2003) or Indo-1AM (Fig. 2).

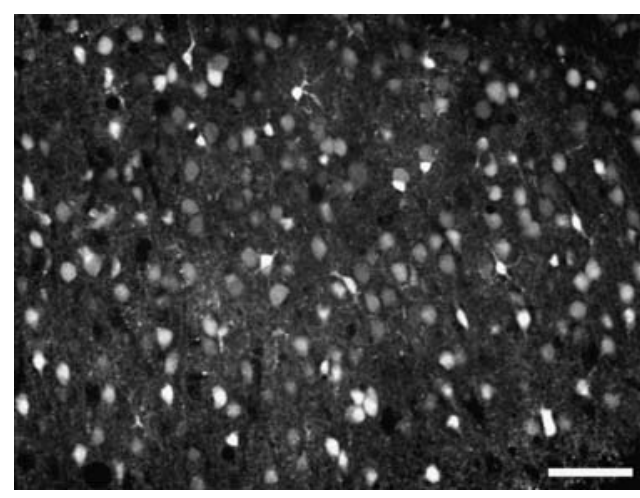

FIGURE 2. Example of imaging. A neocortical brain slice, taken from a postnatal day-13 mouse, loaded with the calcium indicator Indo-1AM. Two-photon fluorescence image acquired with $\sim 730-n m$ excitation wavelength. Scale bar, $50 \mu \mathrm{m}$. 
V. Nikolenko and R. Yuste

\section{REFERENCES}

Cossart R, Aronov D, Yuste R. 2003. Attractor dynamics of network UP states in the neocortex. Nature 423: 283-288.

Majewska A, Yiu G, Yuste R. 2000. A custom-made two-photon microscope and deconvolution system. Pflügers Arch 441: 398-408.

Nikolenko V, Nemet B, Yuste R. 2003. A two-photon and second-harmonic microscope. Methods 30: 3-15.
Tsai PS, Nishimure N, Yoder EJ, Dolnik EM, White GA, Kleinfield D. 2002. Principles, design and construction of a two photon scanning microscope for in vitro and in vivo studies. In Methods for in vivo optical imaging (ed. Frostig R.), pp. 113-171. CRC, New York. 


\section{How to Build a Two-Photon Microscope with a Confocal Scan Head}

Volodymyr Nikolenko and Rafael Yuste

Cold Spring Harb Protoc; doi: 10.1101/pdb.ip075135

\begin{tabular}{rc}
\hline $\begin{array}{r}\text { Email Alerting } \\
\text { Service }\end{array}$ & Receive free email alerts when new articles cite this article - click here. \\
\hline $\begin{array}{c}\text { Subject } \\
\text { Categories }\end{array}$ & $\begin{array}{c}\text { Browse articles on similar topics from Cold Spring Harbor Protocols. } \\
\text { Confocal Microscopy (114 articles) } \\
\text { Imaging/Microscopy, general (579 articles) } \\
\text { Multi-Photon Microscopy (103 articles) }\end{array}$ \\
\hline
\end{tabular}

